REGARDS

SUR L'ECONOMIE ALLEMANDE

BULLETIN ECONOMIQUE DU CRAC

\section{Regards sur l'économie allemande}

Bulletin économique du CIRAC

115 | 2014

Varia

\title{
Pour soutenir la croissance : améliorer le cadre des activités
}

Isabelle Bourgeois

\section{(2) OpenEdition}

1 Journals

Édition électronique

URL : http://journals.openedition.org/rea/4761

DOI : 10.4000/rea.4761

ISSN : 1965-0787

Éditeur

CIRAC

Édition imprimée

Date de publication : 1 décembre 2014

Pagination : $3-4$

ISSN : 1156-8992

\section{Référence électronique}

Isabelle Bourgeois, «Pour soutenir la croissance : améliorer le cadre des activités 》, Regards sur

l'économie allemande [En ligne], 115 | décembre 2014, mis en ligne le 01 décembre 2014, consulté le 15 septembre 2020. URL : http://journals.openedition.org/rea/4761 


\section{Pour soutenir la croissance : améliorer le cadre des activités}

Quoi qu'on en dise, l'économie allemande joue toujours son rôle de locomotive au sein de l'UE, bien qu'elle se soit nettement ralentie depuis le printemps. Alors que l'année avait bien commencé, avec une croissance de $0,8 \%$ au $1^{\mathrm{er}}$ trimestre, le PIB a brusquement reculé de $0,1 \%$ au deuxième, avant de ne progresser que d'un faible $0,1 \%$ au troisième. Pour le quatrième, la Bundesbank s'attend à une hausse de $0,1 \%$ seulement, suivie de $0,2 \%$ au $1^{\text {er }}$ trimestre 2015 . Cet automne, la plupart des prévisions ont été revues à la baisse ; elles sont proches de celles publiées à la minovembre par le Conseil des Sages dans leur rapport annuel : 1,2\% en $2014(1,4 \%$ selon la Bundesbank) et 1,0\% en 2015.

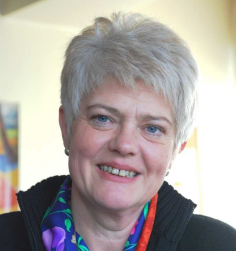

Isabelle Bourgeois, Rédactrice en chef

\begin{tabular}{|c|c|c|c|c|}
\hline \multicolumn{5}{|c|}{ Prévisions du Conseil des Sages } \\
\hline & 2012 & 2013 & 2014 P & $2015 P$ \\
\hline \multicolumn{5}{|l|}{ évolution } \\
\hline PIB (\%) & 0,4 & 0,1 & 1,2 & 1,0 \\
\hline Consommation (\%) & 0,8 & 0,8 & 0,9 & 1,4 \\
\hline privée (\%) & 0,7 & 0,8 & 0,8 & 1,5 \\
\hline publique (\%) & 1,2 & 0,7 & 1,0 & 1,2 \\
\hline \multicolumn{5}{|l|}{ Investissements } \\
\hline biens d'équipement (\%) & $-2,9$ & $-2,7$ & 3,0 & 3,9 \\
\hline construction (\%) & 0,6 & $-0,1$ & 3,5 & 2,0 \\
\hline Demande intérieure (\%) & $-0,9$ & 0,7 & 1,5 & 1,5 \\
\hline Part de l'excédent commercial dans le PIB (points de pourcentage) & 1,3 & $-0,5$ & $-0,2$ & $-0,4$ \\
\hline exportations (\%) & 2,8 & 1,6 & 2,9 & 3,6 \\
\hline importations (\%) & $-0,0$ & 3,1 & 3,7 & 5,1 \\
\hline Prix à la consommation & 2,0 & 1,5 & 1,0 & 1,3 \\
\hline \multicolumn{5}{|l|}{ état } \\
\hline Actifs occupés (milliers) & 42033 & 42281 & 42641 & 42795 \\
\hline Actifs occupant un emploi soumis à cotisations sociales (milliers) & 29341 & 29713 & 30180 & 30474 \\
\hline Chômeurs indemnisés (milliers) & 2897 & 2950 & 2908 & 2931 \\
\hline Taux de chômage (Agence fédérale pour l'emploi) (\%) & 6,8 & 6,9 & 6,7 & 6,7 \\
\hline Déficit public (\% du PIB) & 0,1 & 0,1 & 0,3 & $-0,0$ \\
\hline
\end{tabular}

Source des données : Sachverständigenrat zur Begutachtung der gesamtwirtschaftlichen Entwicklung, Jahresgutachten 2014.

"Il est fondé de penser que la faiblesse conjoncturelle actuelle va s'avérer passagère ». C'est en ces termes que Jens Weidmann, Président de la Bundesbank, présentait les prévisions semestrielles de son institution le 05-12-2014. L'embellie conjoncturelle qui s'est esquissée cet automne et devrait être soutenue dans les prochains mois grâce à un regain d'activité sur le marché mondial et au sein de la zone Euro tirera à nouveau une croissance allemande. Celle-ci avait pâti au printemps de la faiblesse de la demande dans la zone Euro et d'incertitudes géopolitiques pesant sur la balance commerciale, notamment la crise ukrainienne. Cette analyse est partagée par les Instituts économiques qui ont publié leur rapport d'automne en octobre, ainsi que par le Conseil des Sages ou la Bundesbank. Mais depuis, la forte chute des prix du pétrole " agit comme un programme conjoncturel »: elle réduit le coût de la vie pour les ménages et les coûts de production pour les entreprises, résume J. Weidmann qui rappelle la « solidité » des fondamentaux de l'économie allemande.

Le marché du travail est très dynamique et tire la consommation. Depuis janvier 2014, le nombre d'actifs occupés s'est accru d'un million (Destatis) ; celui des chômeurs enregistrés à l'Agence fédérale pour l'emploi continue de baisser, s'installant au-dessous de la barre des 3 millions. Mais si, comme Destatis, on se base sur les critères de I'OIT, le nombre de demandeurs d'emploi est désormais inférieur à 2 millions, et le taux de chômage n'est que de 4,9\%. Les antennes locales de l'Agence fédérale pour l'emploi enregistrent une hausse des demandes de recrutement pour les trois mois à venir, aussi bien dans l'intérim qu'en CDI. Cette tendance prolonge l'évolution de l'année 2013, qui s'était soldée par 4,9 millions de nouvelles embauches ; il s'agissait surtout pour les entreprises de remplacer des départs (38\%) ou de couvrir de nouveaux besoins (50\%) - et ce, sur le long terme (IAB). La demande est pour l'instant couverte par les mouvements migratoires au sein de l'UE. Mais le manque de main-
La faiblesse conjoncturelle de l'été était passagère

Un marché du travail robuste 
Production et échanges : régime de croisière

La stabilité budgétaire entretient la confiance d'œuvre qualifiée et expérimentée commence à se faire sentir, sous l'effet du vieillissement démographique et de l'abaissement de l'âge légal de départ à la retraite à 63 ans pour ceux qui ont cotisé 45 ans à dater du $1^{\mathrm{er}}$ janvier 2015 (voir dans ce numéro).

Mieux que la hausse de l'indice ifo, cette robustesse du marché de l'emploi montre que les entreprises ont regagné confiance en l'avenir après une brève déprime face aux incertitudes mondiales. II est vrai que la reprise de la demande extérieure et l'excellente tenue des commandes les y incitent : en octobre, ces dernières ont progressé de 2,5\% dans l'ensemble (Destatis) - et même de 16,7\% dans l'industrie automobile. La production industrielle se stabilise dès lors après le ralentissement des derniers mois. Après le fléchissement de cet été, les exportations ont elles aussi rebondi au $3^{\mathrm{e}}$ trimestre. La balance commerciale, avec un excédent de 0,2 point de pourcentage au $3^{\mathrm{e}}$ trimestre, contribue ainsi à nouveau à la croissance.

La confiance des consommateurs comme des entreprises est entretenue aussi par la consolidation des finances publiques. Le Bundestag a voté à la fin novembre le premier budget fédéral à l'équilibre depuis... 1969. Quant au niveau de la dette, il est prévu de le ramener de 75,4\% cette année à $70 \%$ du PIB d'ici 2017 , aussi pour mieux respecter la « règle d'or » interne à l'Allemagne qui interdira aux Länder tout recours au déficit dès le $1^{\text {er }}$ janvier 2020. La bonne tenue des finances publiques est facilitée par un contexte favorable : la robustesse du marché du travail comme de l'activité accroît l'encours des prélèvements sociaux et fiscaux, et le chômage ne grève guère les budgets publics ou sociaux. Quant au taux d'intérêt, à un niveau historiquement bas, ils permettent de recourir à la dette - à dose raisonnable toutefois.

L'Allemagne n'est pas guettée par la récession, contrairement à ce que laissaient entendre, au début de l'automne, certains observateurs étrangers. Et elle n'a pas besoin non plus de plan de relance conjoncturel financé à crédit. Ce serait faire fausse route, comme le déclarait on ne peut plus explicitement Sigmar Gabriel (SPD), ministre fédéral de l'Economie, lors de la présentation des prévisions du gouvernement fédéral: "Accroître l'endettement de l'Allemagne n'aurait aucun effet positif sur la croissance en Italie, en France, en Espagne ou en Grèce » (F.A.Z., 14-10-2014).

TOUT IRAIT POUR LE MIEUX DU MONDE SI le gouvernement de grande coalition ne créait pas lui-même une série d'incertitudes quant aux perspectives de croissance. C'est là l'autre raison qui explique que la conjoncture allemande ne tourne qu'à sa vitesse de croisière : «La politique économique est rattrapée par la réalité ». C'est sur elle que porte la critique constructive du rapport annuel 2014 du Conseil des Sages, et dont le titre revendique "Plus de confiance dans le fonctionnement du marché ». Partant de l'idée répandue d'une "prétendue faiblesse des investissements", il rappelle que celle, réelle, de l'investissement public, doit "inciter à reconsidérer les priorités » des dépenses publiques plutôt qu'à "réclamer une hausse des impôts ou de la dette". Quant à une " faiblesse pathologique de l'investissement privé à laquelle il conviendrait d'appliquer un traitement de politique économique ", elle est de pure conjecture.

Car ce qui retient les entreprises, c'est un cadre réservé aux activités qui est tout sauf propice à l'investissement. Le Conseil des Sages critique vertement la politique à contresens que mène la grande coalition : au lieu de consolider d'abord les bases de la croissance, elle redistribue des richesses dont rien ne dit qu'elles seront créées un jour. Les griefs sont nombreux. Au lieu de continuer à lever les rigidités du marché du travail, la grande coalition en crée de nouvelles avec le SMIC légal généralisé qui entrera en vigueur le $1^{\text {er }}$ janvier 2015. Au lieu de pérenniser le financement des régimes de protection sociale dans une société vieillissante, elle gaspille les ressources: retraite à 63 ans, baisse de 0,2 point du taux de cotisation retraite qui sera de 18,7\% en 2015... Sa politique de transition énergétique doit être révisée dans l'objectif de gains d'efficience. S'ajoute à cela l'éternelle question du poids désincitatif des prélèvements fiscaux. Enfin, en matière de finances publiques, il convient de réformer l'organisation du fédéralisme financier pour déboucher sur une "Constitution financière activante ", c'est-à-dire non seulement durable mais plus propice à l'innovation et à l'investissement. Mais surtout, il ne faut pas céder à la tentation du relâchement budgétaire : en effet, "dans la zone Euro, la crédibilité des règles budgétaires [étant] loin d'être garantie.... le gouvernement fédéral doit donner l'exemple ». Cela vaut aussi bien pour la stabilité budgétaire que pour les réformes de structure. C'est là, parmi tant d'autres, un appel pressant à l'Allemagne à ne pas se reposer sur ses lauriers. (I. B., 09/12/2014) 